\title{
PSEUDOSPIN-ELECTRON MODEL IN INFINITE DIMENSIONS
}

\author{
I. V. Stasyuk, A. M. Shvaika \\ Institute for Condensed Matter Physics, National Acadamy of Sciences of the Ukraine, \\ 1 Svientsitskii Str., Lviv, UA-290011, Ukraine
}

(Received March 11, 1999)

\begin{abstract}
Energy spectrum and thermodynamics of the pseudospin-electron model are investigated in the dynamical mean field approximation ( $d=\infty$ limit). In the limit of zero electron correlation this model is analytically exactly soluble within this approach: in the $\mu=$ const regime the first order phase transition with the jump of the pseudospin mean value and reconstruction of the electron spectrum can be realized, while in the $n=$ const regime the phase separation in electron subsystem can take place for certain values of the model parameters. On the basis of the obtained results the applicability of the approximate schemes previously used for the investigation of the pseudospinelectron model are discussed.

Key words: pseudospin-electron model, Falicov-Kimball model, dynamical mean field theory, phase transition, phase separation.
\end{abstract}

PACS numbers: 71.10.Fd, 71.38.+i, 77.80.Bh, 63.20.Ry

\section{INTRODUCTION}

The theoretical investigation of the strongly correlated electron systems is an enduring subject of interest in condensed matter physics especially after the discovery of high- $T_{c}$ superconductivity.

In recent years the essential achievements of the theory of strongly correlated electron systems have been connected with the development of the dynamical mean field theory (DMFT) proposed by Metzner and Vollhardt [1] for the Hubbard model (see also [2] and references therein). DMFT is a nonperturbative scheme which allows to project the Hubbard model on the single impurity Anderson's model and is exact in the limit of infinite space dimension $(d=\infty)$. Moreover, some class of the binary alloy type models (e.g. Falicov-Kimball model [3]) can be studied almost analytically within DMFT.

The Hamiltonian of the binary alloy type models can be written in the form:

$$
H=\sum_{i} H_{i}+\sum_{i j \sigma} t_{i j} a_{i \sigma}^{\dagger} a_{j \sigma}
$$

where

$$
H_{i}=g S_{i}^{z} \sum_{\sigma} n_{i \sigma}-\mu \sum_{\sigma} n_{i \sigma}-h S_{i}^{z}
$$

is single-site Hamiltonian, and includes local interaction ( $g$-term) of conducting electrons with some two level subsystem described by pseudospins $S_{i}^{z}= \pm \frac{1}{2}$ placed in the longitudinal field $h$.

Hamiltonian (1.1), (1.2) is a simplified version of the pseudospin-electron model when we neglect electron correlations. In general, pseudospin-electron model [4] was proposed to be included into the theory the interaction of correlated electrons with some local lattice excitations described by pseudospins (e.g. anharmonic vibrations of apex oxygen in YBaCuO type HTSC's). It shows the possibility of dipole (pseudospin) and charge density instabilities [5,6] and phase separation [7] due to the effective retarded interaction between pseudospins via conducting electrons. All these results were obtained within the generalized random phase approximation (GRPA) [8] which is a realization of the appropriate perturbation theory for correlation functions in the case of strong coupling $(U \gg t)$ and corresponds to the mean field type approximation in the calculation of mean values.

It is convenient to introduce projective operators on pseudospin states

$$
P_{i}^{ \pm}=\frac{1}{2} \pm S_{i}^{z}, \quad\left(P_{i}^{ \pm}\right)^{2}=P_{i}^{ \pm}, \quad P_{i}^{+} P_{i}^{-}=0
$$

and Hamiltonian of the binary alloy can be obtained by substitution $P_{i}^{+}=c_{i}, P_{i}^{-}=1-c_{i}$, where $c_{i}$ is the concentration of one component of binary alloy and $1-c_{i}$ is the concentration of the second one. On the other hand, if we remove in (1.1) and (1.2) spin indices and rewrite Hamiltonian in terms of the operators of the mobile $d_{i}$ $\left(a_{i \sigma}=d_{i}\right)$ and localized $f_{i}\left(P_{i}^{+}=f_{i}^{\dagger} f_{i}, P_{i}^{-}=1-f_{i}^{\dagger} f_{i}\right)$ electrons we shall get the Hamiltonian of the FalicovKimball model where $h$ plays a role of the chemical potential for the localized electrons (or ions). As a rule the common chemical potential is introduced for both electron subsystems but the case of two chemical potentials was also considered (see, e.g. [9]) and the first consideration of Falicov-Kimball model within DMFT was done by Brandt and Mielsch [3].

The main difference between these models is in the way how an averaging procedure over projection operators is performed (thermal statistical averaging in the case of pseudospin-electron and Falicov-Kimball models and configurational averaging for binary alloy) and how self-consistency is achieved (fixed value of longitu- 
dinal field $h$ for pseudospin-electron model, fixed value of the component concentration $c$ for binary alloy and fixed value of the electron concentration - total or for both electron subsystems - for Falicov-Kimball model).

The ground state of the Falicov-Kimball model, when the electron concentration for subsystems is fixed (the fixed values of the electron concentration and pseudospin mean value for pseudospin-electron model), is not uniform and shows the commensurate or incommensurate ordering or phase separation depending on the concentration and coupling constant values [9]. On the other hand, in the case of the fixed value of the longitudinal field $h$ the possibility of the jump-like uniform changes of the pseudospin mean value without symmetry break (bistability) appears. Such bistability is caused by the effective many-body interactions between pseudospins vs. conducting electrons.

Here we investigate the possibility of such phase transitions without symmetry break for pseudospin-electron model described by the Hamiltonian (1.1) within DMFT, which exactly takes into account all single-site manybody interactions.

\section{PERTURBATION THEORY IN TERMS OF ELECTRON TRANSFER AND THE LIMIT OF LARGE DIMENSIONS $(d \rightarrow \infty)$}

In general, one-electron Green's function $G_{\sigma}\left(\omega_{n}, \mathbf{k}\right)$

$$
G_{i j}^{\sigma}\left(\tau-\tau^{\prime}\right)=\left\langle\mathcal{T} a_{i \sigma}(\tau) a_{j \sigma}^{\dagger}\left(\tau^{\prime}\right) \sigma(\beta)\right\rangle_{0} /\langle\sigma(\beta)\rangle_{0}
$$

where scattering matrix is determined by

$$
\sigma(\beta)=\mathcal{T} \exp \left\{-\int_{0}^{\beta} d \tau \sum_{i j \sigma} t_{i j} a_{i \sigma}^{\dagger}(\tau) a_{j \sigma}(\tau)\right\}
$$

$\beta=1 / T$ and averaging $\langle\ldots\rangle_{0}$ is performed with the one site part $H_{0}=\sum_{i} H_{i}$ of the Hamiltonian (1.1), satisfies Larkin's equation

$$
\begin{aligned}
G_{i j}^{\sigma}\left(\tau-\tau^{\prime}\right) & =\Xi_{i j}^{\sigma}\left(\tau-\tau^{\prime}\right) \\
& +\Xi_{i l}^{\sigma}\left(\tau-\tau^{\prime \prime}\right) t_{l m} G_{m j}^{\sigma}\left(\tau^{\prime \prime}-\tau^{\prime}\right)
\end{aligned}
$$

Here $\Xi_{i j}^{\sigma}\left(\tau-\tau^{\prime}\right)$ is the total irreducible according to Larkin part [10] and summation (integration) over repeated indices is supposed. The formal solution of Eq. (2.3) can be written in the form

$$
G_{\sigma}\left(\omega_{n}, \mathbf{k}\right)=\frac{1}{\Xi_{\sigma}^{-1}\left(\omega_{n}, \mathbf{k}\right)-t_{\mathbf{k}}}
$$

and the problem is to calculate the irreducible parts $\Xi_{\sigma}\left(\omega_{n}, \mathbf{k}\right)$.

Formally diagrammatic series for the irreducible part $\Xi_{i j}^{\sigma}\left(\omega_{n}\right)$ are the same for all these models

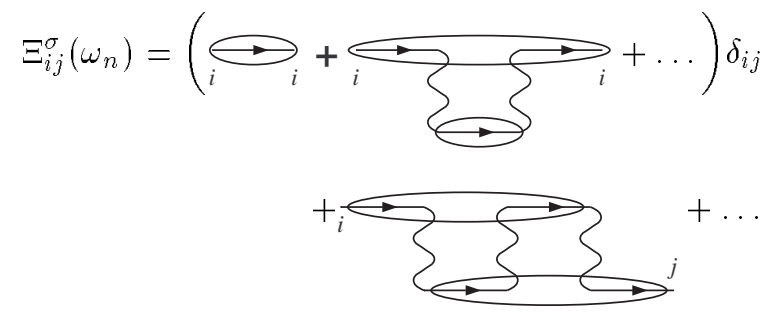

and includes both single-site and intersite contributions. Here, arrows indicate electron propagators $g_{\sigma i}^{ \pm}\left(\omega_{n}\right)=$ $\frac{P_{i}^{ \pm}}{i \omega_{n}+\mu \mp \frac{g}{2}}$ in the subspaces projected on the pseudospin states of site $i$ and ovals represent semi-invariant averaging of projection operators.

In the case of high dimensions $(d \rightarrow \infty)$ one should scale hopping integral

$$
t_{i j} \rightarrow \frac{t_{i j}}{\sqrt{d}}
$$

in order to obtain finite density of states (the Gaussian one for $d=\infty$ hypercubic lattice $\rho(\varepsilon)=\frac{1}{W \sqrt{\pi}} e^{-\varepsilon^{2} / W^{2}}$ and semi-elliptic DOS for $d=\infty$ Bethe lattice $\rho(\varepsilon)=$ $\left.\frac{2}{\pi W^{2}} \sqrt{W^{2}-\varepsilon^{2}}[2]\right)$. Due to such scaling only single-site contributions survive in the expression for irreducible parts $\Xi_{\sigma}[11]$

$$
\Xi_{i j}^{\sigma}\left(\tau-\tau^{\prime}\right)=\delta_{i j} \Xi_{\sigma}\left(\tau-\tau^{\prime}\right), \Xi_{\sigma}\left(\omega_{n}, \mathbf{k}\right)=\Xi_{\sigma}\left(\omega_{n}\right)
$$

and such a site-diagonal function, as shown by Brandt and Mielsch [3], can be calculated by mapping the infinite-dimensional lattice problem on the atomic model

$$
\begin{aligned}
& e^{-\beta H} \rightarrow e^{-\beta H_{\mathrm{eff}}}=e^{-\beta H_{0}} \\
& \times \mathcal{T} \exp \left\{-\int_{0}^{\beta} d \tau \int_{0}^{\beta} d \tau^{\prime} \sum_{\sigma} J_{\sigma}\left(\tau-\tau^{\prime}\right) a_{\sigma}^{\dagger}(\tau) a_{\sigma}\left(\tau^{\prime}\right)\right\}
\end{aligned}
$$

with auxiliary Kadanoff-Baym field $J_{\sigma}\left(\tau-\tau^{\prime}\right)$ [12] which has to be selfconsistently determined from the condition that the same function $\Xi_{\sigma}$ defines Green's functions for lattice (2.4) and atomic limit

$$
G_{\sigma}^{(a)}\left(\omega_{n}\right)=\frac{1}{\Xi_{\sigma}^{-1}\left(\omega_{n}\right)-J_{\sigma}\left(\omega_{n}\right)}
$$

"Dynamical" mean field $J_{\sigma}\left(\tau-\tau^{\prime}\right)$ describes the hopping (transfer) of electron from the atom into the environment at the moment $\tau$, propagation in the environment without stray into the atom until the moment $\tau^{\prime}$. Connection between these "dynamical" mean field of atomic problem and Green's function of the lattice can be obtained using a standard CPA approach [2]:

$$
J_{\sigma}\left(\omega_{n}\right)=\Xi_{\sigma}^{-1}\left(\omega_{n}\right)-G_{\sigma}^{-1}\left(\omega_{n}\right),
$$


where

$$
G_{\sigma}^{(a)}\left(\omega_{n}\right)=G_{\sigma}\left(\omega_{n}\right)=\int_{-\infty}^{+\infty} d t \frac{\rho(t)}{\Xi_{\sigma}^{-1}\left(\omega_{n}\right)-t}
$$

is a single-site Green's function both for atomic limit and lattice. Here summation over wave vector was changed by the integration with the density of states $\rho(t)$.

In order to complete our self-consistent set of equations we should find expression for Green's function in the atomic limit (2.9). Due to the properties of the projection operators (1.3) one can rewrite Hamiltonian of atomic problem (2.8) in the form

$$
e^{-\beta H_{\text {eff }}}=P^{+} e^{-\beta H_{+}}+P^{-} e^{-\beta H_{-}}
$$

and our space of states splits into two independent subspaces hence all projection operators (1.3) act at the same site and in any order of the perturbation theory expansion all projection operators can be replaced by their product result and there are no necessity to make semi-invariant expansions.

Single-electron Green's function is a sum of Green's functions in subspaces and is equal

$$
\begin{aligned}
G_{\sigma}^{(a)}\left(\omega_{n}\right) & =\frac{\left\langle P^{+}\right\rangle}{i \omega_{n}+\mu-J_{\sigma}\left(\omega_{n}\right)-\frac{g}{2}} \\
& +\frac{\left\langle P^{-}\right\rangle}{i \omega_{n}+\mu-J_{\sigma}\left(\omega_{n}\right)+\frac{g}{2}}
\end{aligned}
$$

Here $\langle\ldots\rangle$ is a statistical averaging with the effective Hamiltonian (2.8). For the one-site functions it is equivalent to the averaging with the total Hamiltonian (1.1).

Partition functions in subspaces are

$$
\begin{aligned}
& Z_{ \pm}=\operatorname{Sp} e^{-\beta H_{ \pm}}=e^{ \pm \frac{\beta h}{2}-Q_{ \pm}} \\
& =e^{ \pm \frac{\beta h}{2}} \prod_{\sigma}\left(1+e^{\beta\left(\mu \mp \frac{g}{2}\right)}\right) \prod_{n}\left(1-\frac{J_{\sigma}\left(\omega_{n}\right)}{i \omega_{n}+\mu \mp \frac{g}{2}}\right)
\end{aligned}
$$

and presents the partition functions of the non-interacting fermions with frequency dependent hopping placed in the external field formed by pseudospin.

Pseudospin mean value is determined by equation

$$
\begin{aligned}
& \left\langle S^{z}\right\rangle=\frac{1}{2} \frac{Z_{+}-Z_{-}}{Z_{+}+Z_{-}} \\
& =\frac{1}{2} \tanh \frac{1}{2}\left(\beta h-\left(Q_{+}\left[\left\langle S^{z}\right\rangle\right]-Q_{-}\left[\left\langle S^{z}\right\rangle\right]\right)\right)
\end{aligned}
$$

which is an analogue of the well known equation of state for Ising model in mean-field approximation: $\left\langle S^{z}\right\rangle=$ $\frac{1}{2} \tanh \frac{\beta}{2}\left(h+J_{0}\left\langle S^{z}\right\rangle\right)$. It should be noted that in the case of Lorentzian density of states $\rho(\varepsilon)=\frac{W}{\pi\left(W^{2}+\varepsilon^{2}\right)}$, which is frequently used in some applications of DMFT, one can easily obtain a simple result $J_{\sigma}\left(\omega_{n}\right)=i W$ [2], quantities $Q^{ \pm}$do not depend on $\left\langle S^{z}\right\rangle$ and equation (2.15) transforms into an expression for $\left\langle S^{z}\right\rangle$ that indicates the sensitivity of the equation of state to the shape of DOS.

Electron concentration mean value is determined by

$$
\langle n\rangle=\frac{1}{\beta} \sum_{m \sigma} G_{\sigma}\left(\omega_{m}\right)
$$

and the functional of thermodynamic potential can be derived in the same way as it was done in [3] for FalicovKimball model

$$
\frac{\Omega}{N}=\Omega_{(a)}-\frac{1}{\beta} \sum_{n \sigma}\left\{\ln G_{\sigma}^{(a)}\left(\omega_{n}\right)-\frac{1}{N} \sum_{\mathbf{k}} \ln G_{\sigma}\left(\omega_{n}, \mathbf{k}\right)\right\},
$$

where

$$
\Omega_{(a)}=-\frac{1}{\beta} \ln \left(Z_{+}+Z_{-}\right)
$$

is a thermodynamic potential for the atomic problem.

Below, all calculations will be performed for semielliptic DOS when the auxiliary field is determined by the simple cubic equation

$$
\begin{array}{r}
J_{\sigma}\left(\omega_{n}\right)=\frac{W^{2}}{4}\left\{\frac{\left\langle P^{+}\right\rangle}{i \omega_{n}+\mu-J_{\sigma}\left(\omega_{n}\right)-\frac{g}{2}}\right. \\
\left.+\frac{\left\langle P^{-}\right\rangle}{i \omega_{n}+\mu-J_{\sigma}\left(\omega_{n}\right)+\frac{g}{2}}\right\} .
\end{array}
$$

In a usual way we perform analytical continuation on real axis $\left(i \omega_{n} \rightarrow \omega-i \delta\right)$ and the only solutions of (2.19) with $\Im m J_{\sigma}(\omega)>0$ must be considered. Band boundaries are determined from the condition $\Im m J_{\sigma}(\omega) \rightarrow 0$ and in Fig. 1 their dependence on coupling constant $g$ are presented. One can see that there exists critical value of coupling constant $g \sim W$ when a gap in spectrum appears. It should be noted that within GRPA as well as in other approaches where single-electron Green's function is calculated in Hubbard-I approximation, when we keep only the first term of the single-site contribution in the expression for the irreducible part (2.5), this gap in spectrum always exists.

In the case of strong $(g \gg W)$ coupling an analytical solutions can be obtained

$$
J_{\sigma}(\omega)=\frac{1}{2}\left(\omega \mp \frac{g}{2}\right)+\frac{i}{2} \sqrt{W^{2}\left\langle P^{ \pm}\right\rangle-\left(\omega \mp \frac{g}{2}\right)^{2}}
$$

for upper and lower subbands, respectively, and one can see that subbands halfwidth is equal to $W \sqrt{\frac{1}{2} \pm\left\langle S^{z}\right\rangle}$ whereas in Hubbard-I approximation it is $W\left(\frac{1}{2} \pm\left\langle S^{z}\right\rangle\right)$. 
This result clearly shows that even for the case of strong coupling when subbands are well separated and one of them becomes narrow $\left(\left\langle S^{z}\right\rangle \rightarrow \pm \frac{1}{2}\right)$ Hubbard-I approximation is unsufficient and can not be derived from the exact solution in any way, e.g. due to the subbands halfwidth square root dependence on the localized states occupancy $\left(\left\langle P^{\mp}\right\rangle \rightarrow 0\right)$.

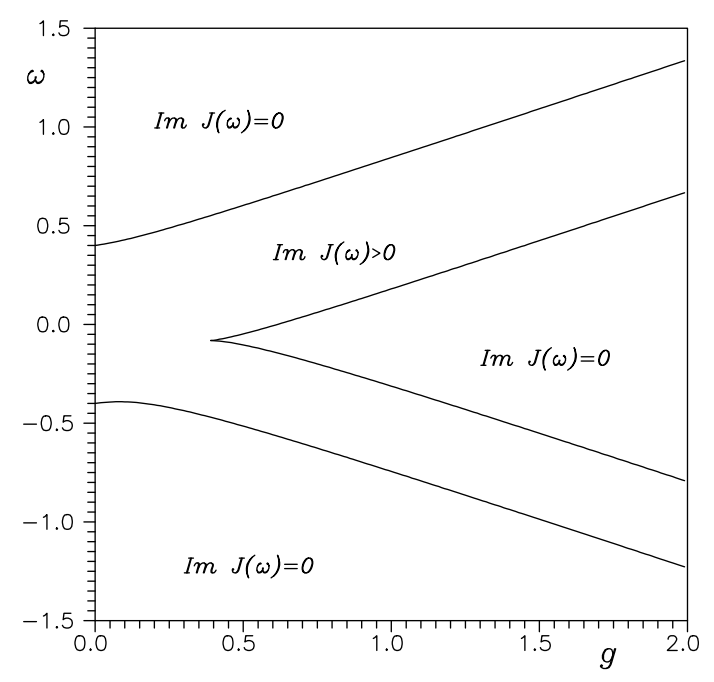

Fig. 1. Electron bands boundaries (semi-elliptic DOS, $\left.W=0.4,\left\langle S^{z}\right\rangle=0.2\right)$.

The expressions presented above were obtained for the fixed value of the chemical potential $\mu$ when stable states are determined from the minimum of the thermodynamical potential (2.17). This regime $\mu=$ const corresponds to the case when the charge redistribution between conducting sheets $\mathrm{CuO}_{2}$ and other structural elements (charge reservoir, e.g. nonstoichiometric in oxygen $\mathrm{CuO}$ chains in $\mathrm{YBaCuO}$ type structures) which fix the value of the chemical potential is allowed. On the other hand, in the regime of the fixed electron concentration value one should solve equation for the chemical potential $n=\langle n\rangle$ (2.16) and stable states are determined by the minimum of the free energy $F=\Omega+\mu n$.

\section{RESULTS AND DISCUSSION}

Integrals in Eqs. (2.14) and (2.17) can be calculated analytically for states with $\left\langle S^{z}\right\rangle= \pm \frac{1}{2}$ at zero temperature and corresponding phase diagrams $\mu-h$ which indicate stability regions for these states are shown in Fig. 2a and b for $g>W$ and $g<W$, respectively. One can see two regions of $\mu$ and $h$ values where the states with $\left\langle S^{z}\right\rangle= \pm \frac{1}{2}$ coexist. In the vicinity of these regions the phase transitions of first order with the change of the longitudinal field $h$ and/or chemical potential $\mu$ take place (see Fig. 3) and they are shown by thick lines on phase diagrams (Fig. 2).

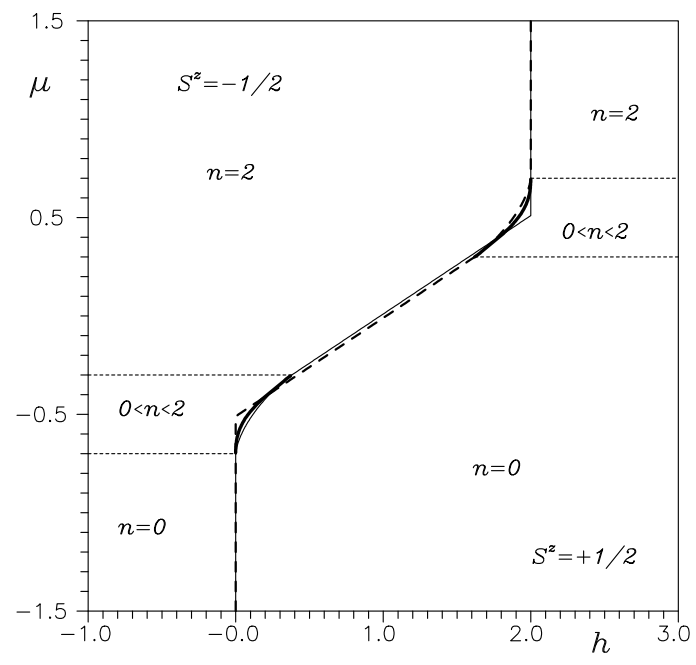

a)

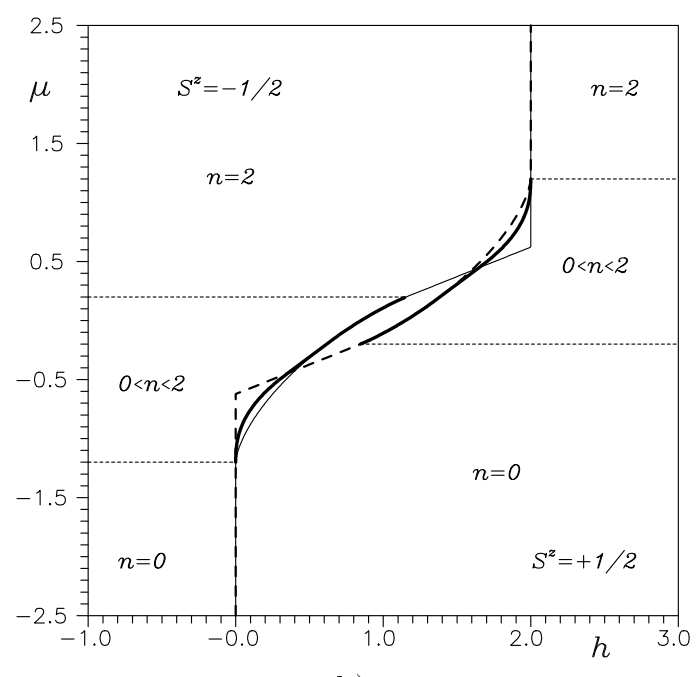

b)

Fig. 2. Phase diagram $\mu-h$. Dashed and thin solid lines surround regions with $S^{z}= \pm \frac{1}{2}$, respectively. Thick solid line indicate the first order phase transition points. a) $g=1$, $W=0.2$; b) $g=1, W=0.7$.

There is not any specific behaviour when chemical potential is placed out of bands. If chemical potential is placed in the upper subband the graphs presented in Fig. 3 transform according to the internal symmetry of the Hamiltonian:

$$
\mu \rightarrow-\mu, \quad h \rightarrow 2 g-h, \quad n \rightarrow 2-n, \quad S^{z} \rightarrow-S^{z} .
$$

With the temperature increase the region of the phase coexistence narrows and the corresponding phase diagram $T_{c}-h$ is shown in Fig. 4 . One can see that with respect to Ising model the phase coexistence curve is shifted in the field and distorted from the vertical line and hence the possibility of the first order phase transition with the temperature change exists in pseudospinelectron model for the narrow range of $h$ values. 


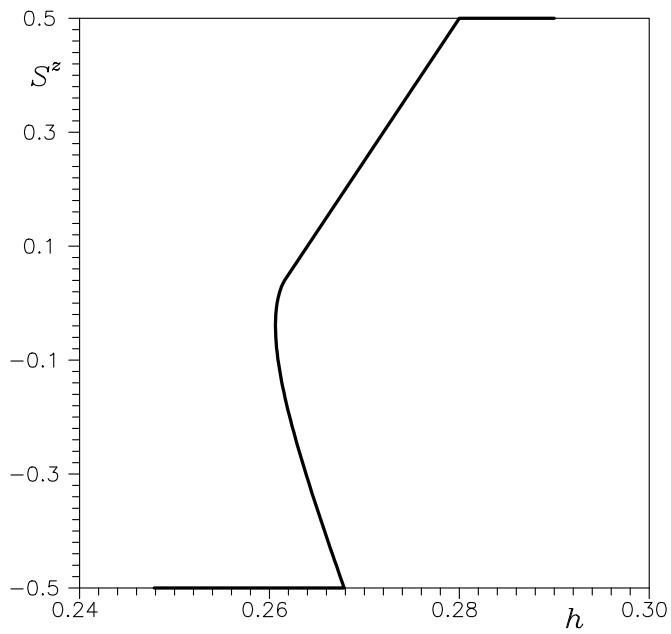

a)

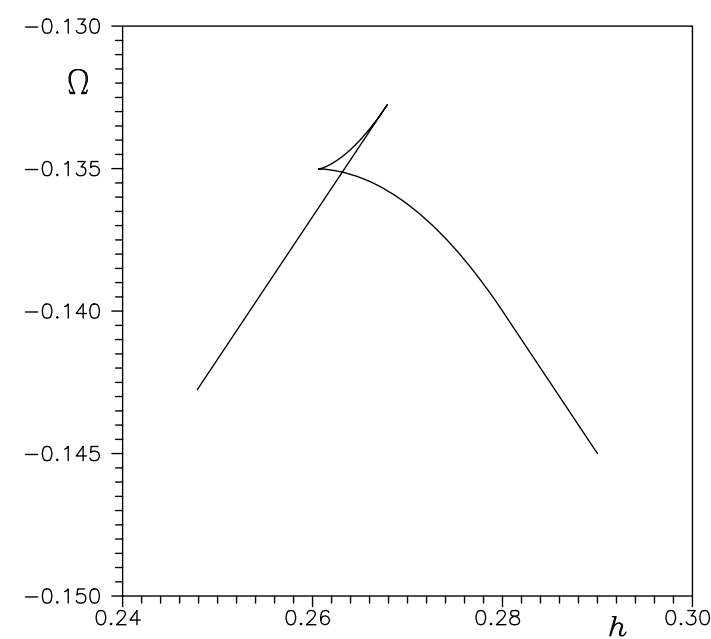

b)

Fig. 3. Field dependencies of $\left\langle S_{z}\right\rangle$ (a) and thermodynamical potential (b) for $\mu=$ const regime when chemical potential is placed in the lower subband $\mu=-0.37$ ( $W=0.2, g=1$, $T=0)$.

As mentioned above, the band structure is determined by the pseudospin mean value and its change is accompanied by the corresponding changes of the electron concentration and for the $(\mu, h)$ values fixed on the Ist order phase transition line there are three solutions for electron concentration one of which is unstable.

In the case of the fixed value of the electron concentration value (regime $n=$ const) this Ist order phase transition transforms into the phase separation. One can see regions with $d \mu / d n \leq 0$, which correspond to this effect in electron subsystem, on the concentration dependencies (Figs. 5 and 6a).

The corresponding dependencies of free energy $F=$ $\Omega+\mu n$ are given in Fig. $6 \mathrm{~b}$. In the phase separated region free energy deflects up and concentration values at binodal points are determined by the tangent line touch points Fig. 6b or from the chemical potential dependencies (Fig. 6a) using Maxwell construction. Resulting phase diagram $T-n$ is shown in Fig. 7 .

For the first time the possibility of phase separation in pseudospin-electron model was marked in [7] where it was obtained within GRPA in the limit of strong correlation $U \rightarrow \infty$. Here it is observed for the opposite case of $U=0$.

The problem of phase separation in strongly correlated systems is not new (see [13] and references therein). It was shown for Hubbard and $t-J$ models [14] that for some parameter values system separates into hole-rich and hole-poor regions with paramagnetic and antiferromagnetic orders, respectively, and long-range interaction between these charged regions is considered as an origin of the appearance of stripe structures.

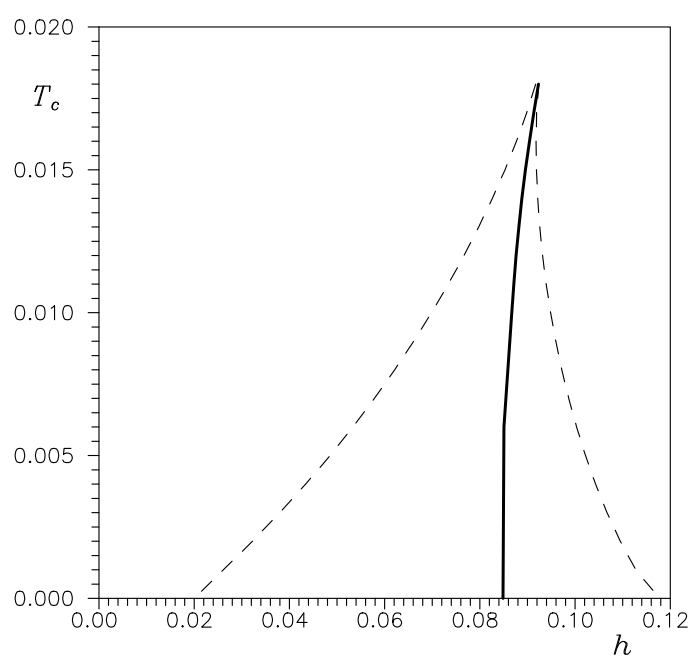

Fig. 4. Phase diagram $T_{c}-h$ : solid and dashed lines indicate the first order phase transition line and boundaries of the phase stability, respectively $(g=1, W=0.2, \mu=-0.5)$

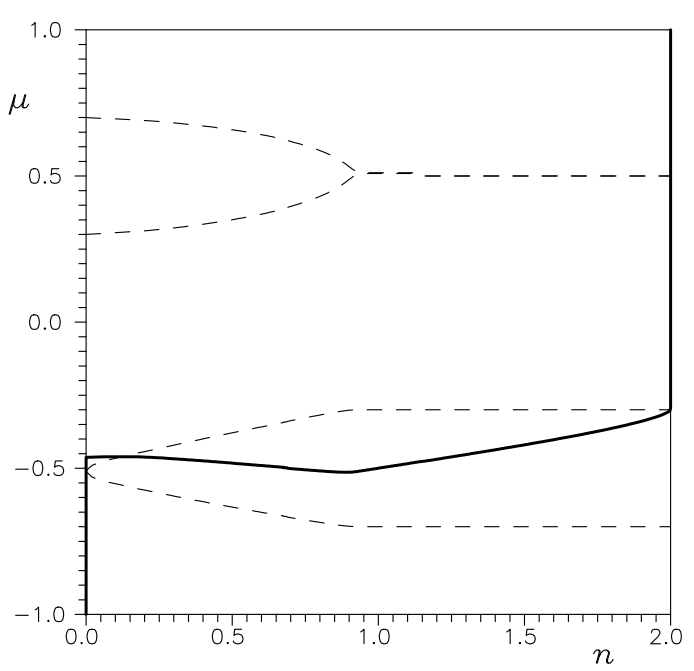

Fig. 5. Dependence of the chemical potential $\mu$ and electron bands boundaries (dashed lines) on the electron concentration $n(T=0.001, g=1, W=0.2, h=0.1)$. 


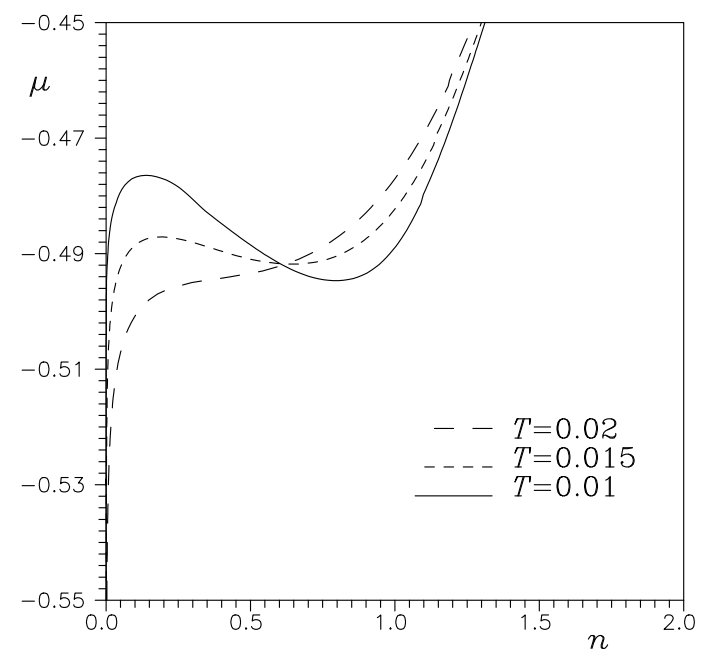

a)

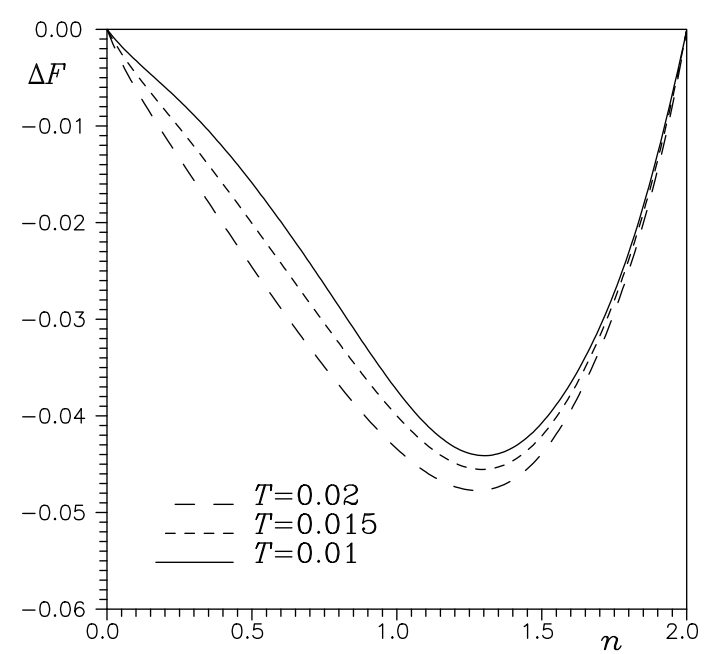

b)

Fig. 6. Dependence of the chemical potential $\mu$ (a) and deviation of free energy from linear dependence $\Delta F=F(n)-\frac{n}{2} F(2)-\left(1-\frac{n}{2}\right) F(0)$ (b) on the electron concentration $n$ for different temperatures $T(g=1, W=0.2$, $h=0.1)$.

In Ref. [9] the phase segregation for some parameter values was reported for the annealed binary alloy with diagonal disorder described by Falicov-Kimball model. In our case of the pseudospin-electron model without electron correlations system separates into regions with different values of electron concentration and pseudospin mean value and electron spectrum contains both wide empty electron band and occupied localized states of the regions with $n \sim 0$ as well as partially filled wide electron band and empty localized states of the regions with $n \sim 1$ (see Fig. 5) the weights of which are determined by the electron concentration.
Localized states of such type (polarons) result from the strong electron-pseudospin (out of plane apical oxygen vibrations) coupling $(g>W)$ in the case of $\mathrm{YBaCuO}-$ type structures and it is supposed that the hopping between such polarons gives significant contribution in the carrier relaxation observed by the resonant Raman spectroscopy [15].

It should be noted that in the case of spinless fermions Hamiltonian (1.1) can be applied for the description of the oxygen vacancies subsystem in high- $T_{C}$ superconductors, which can be treated as quasiequilibrium, and it is known that their interaction with some relaxation type lattice mode leads to the phase separation and appearance of superstructures and stripes [16].

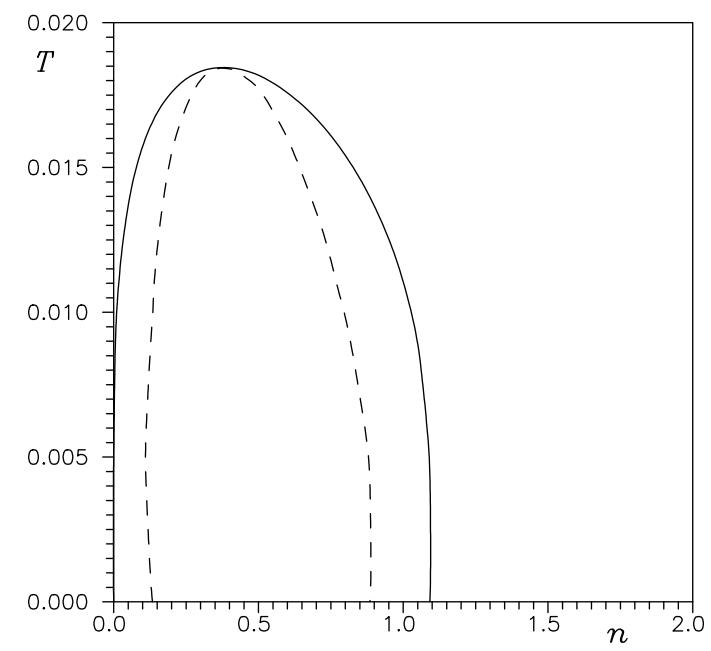

Fig. 7. Phase diagram $T-n$ for phase separated state: solid line - binodal, dashed line - spinodal $(g=1, W=0.2$, $h=0.1)$.

In this paper we investigated the possible phase transitions in pseudospin-electron model within DMFT without creation of super structures $(\mathbf{k}=0)$ and the phase diagrams presented in Figs. 4 and 7 concern only this case. In order to detect instabilities associated with a specific wave vectors one should calculate response functions which will be the subject of the further investigations.

\section{ACKNOWLEDGEMENT}

This work was partially supported by the Ministry of Ukraine for Science and Technology (Project No $2.4 / 171)$. 
[1] W. Metzner, D. Vollhardt, Phys. Rev. Lett. 62, 324 (1989).

[2] A. Georges, G. Kotliar, W. Krauth, M.J. Rosenberg, Rev. Mod. Phys. 68, 13 (1996).

[3] U. Brandt, C. Mielsch, Z. Phys. B 75, 365 (1989); 79, 295 (1990); 82, 37 (1991).

[4] K. A. Müller, Z. Phys. B 80, 193 (1990).

[5] I. V. Stasyuk, A. M. Shvaika, Physica C 235-240, 2173 (1994); Cond. Matt. Phys. (Lviv) iss. 3, 134 (1994).

[6] I. V. Stasyuk, A. M. Shvaika, O. D. Danyliv, Mol. Phys. Rep. 9, 61 (1995).

[7] I. V. Stasyuk, A. M. Shvaika, Czech. J. Phys. 46, 961 (1996).

[8] Yu. A. Izyumov, B. M. Letfulov, E. V. Shipitsyn, M. Bartkowiak, K. A. Chao, Phys. Rev. B 46, 15697 (1992).
[9] J. K. Freericks, Phys. Rev. B 47, 9263 (1993).

[10] V. G. Bar'yakhtar, V. V. Krivoruchko, D. A. Yablonskii, Funktsii Grina v tieorii magnietizma (Green's functions in theory of magnetism) (Naukova dumka, Kyiv, 1984).

[11] W. Metzner, Phys. Rev. B. 43, 8549 (1991).

[12] G. Baym, L. P. Kadanoff, Phys. Rev. 124, 287 (1961); G. Baym, Phys. Rev. 127, 1391 (1962).

[13] E. Dagotto, Rev. Mod. Phys. 66, 763 (1994).

[14] V. J. Emery, S. A. Kivelson, Physica C 209, 597 (1993); U. Löw, V. J. Emery, K. Fabricius, S. A. Kivelson, Phys. Rev. Lett. 72, 1918 (1994).

[15] T. Mertelj, J. Demsar, B. Podobnik, I. Poberaj, D. Mihailovic, Phys. Rev. B 55, 6061 (1997).

[16] A. A. Aligia, J. Garćes, J. P. Abriata, Physica C 221, 109 (1994).

\section{ПСЕВДОСПІН-ЕЛ ЕКТРОННА МОДЕЛЬ ПРИ БЕЗМЕЖНІЙ ВИМІРНОСТІ ПРОСТОРУ}

I. В. Стасюк, А. М. Швайка

Інститут фізики коденсованих систем Національной академіӥнаук Украӥни, вул. Свенціцького, 1, 290011, Львіб, Украйна

\footnotetext{
У набли женні динамічного середнього поля (границя $d=\infty$ ) розглянуто енергетичний спектр та термодинаміку псевдоспін-електронної моделі, яка була введена при вивченні ангармонічних ефектів у високотемпературних надпровідниках. У границі нульової електронної кореляції ця модель є точно розв'язуваною в рамках цього підходу: у режимі $\mu=$ const має місце фазовий перехід першого роду зі стрибком середнього значення псевдоспіну і перебудовоюелектронного спектра, тоді як у режимі $n=$ const при певних значеннях параметрів може відбутися фазове розшарування в електронній підсистемі. На основі отриманих результатів обговорено межі застосовности наближених підходів, які раніше використовували при дослідженні псевдоспін-електронної моделі.
} 